\title{
UNLIMITED SAMPLING OF SPARSE SIGNALS
}

\author{
Ayush Bhandari ${ }^{\dagger}$, Felix Krahmer ${ }^{\ddagger}$ and Ramesh Raskar ${ }^{\dagger}$ \\ ${ }^{\dagger}$ Massachusetts Institute of Technology, 77 Mass. Ave. Cambridge 02139, MA, USA. \\ ${ }^{\ddagger}$ Technical University of Munich, Boltzmannstraße 3, 85747 Garching/Munich, Germany. \\ Email: ayush@mit.edu • felix.krahmer@tum.de • raskar@mit.edu
}

\begin{abstract}
In a recent paper [1], we introduced the concept of "Unlimited Sampling". This unique approach circumvents the clipping or saturation problem in conventional analog-to-digital converters (ADCs) by considering a radically different ADC architecture which resets the input voltage before saturation. Such ADCs, also known as Self-Reset ADCs (SR-ADCs), allow for sensing modulo samples. In analogy to Shannon's sampling theorem, the unlimited sampling theorem proves that a bandlimited signal can be recovered from modulo samples provided that a certain sampling density criterion, that is independent of the ADC threshold, is satisfied. In this way, our result allows for perfect recovery of a bandlimited function whose amplitude exceeds the ADC threshold by orders of magnitude. By capitalizing on this result, in this paper, we consider the inverse problem of recovering a sparse signal from its low-pass filtered version. This problem frequently arises in several areas of science and engineering and in context of signal processing, it is studied in several flavors, namely, sparse or FRI sampling, super-resolution and sparse deconvolution. By considering the SR-ADC architecture, we develop a sampling theory for modulo sampling of low-pass filtered spikes. Our main result consists of a new sparse sampling theorem and an algorithm which stably recovers a $K$-sparse signal from low-pass, modulo samples. We validate our results using numerical experiments.
\end{abstract}

\section{INTRODUCTION}

\subsection{Sampling and Recovery of Sparse Signals in Theory}

Recovering spikes from low-pass filtered measurements is a problem that finds applications in several fields of science and engineering. Concretely speaking, consider the model:

$$
g(t)=\sum_{k=0}^{K-1} c_{k} \psi\left(t-t_{k}\right) \equiv\left(s_{K} * \psi\right)(t)
$$

where $\psi$ is a bandlimited function and $s_{K}$ is a continuous time, $K$-sparse, $\tau$-periodic signal,

$$
s_{K}(t)=\sum_{m \in \mathbb{Z}} \sum_{k=0}^{K-1} c_{k} \delta\left(t-t_{k}-m \tau\right), \quad t_{k+1}>t_{k} .
$$

With $\psi$ known and given sampled measurements $y_{n}=y(n T), n=0, \ldots, N-1$, where $T>0$ is the sampling rate, one is typically interested in recovering $s_{K}(t)$ from discrete set of $N$ measurements $\left\{g_{n}\right\}_{n=0}^{N-1}$. In the recent years, this problem has been widely studied under the theme of (a) sparse deconvolution [2], (b) sparse or FRI sampling [3,4] and (c) super-resolution [5]. While this problem has a known history with roots tracing back to seismic imaging [6,7], recent developments allow for recovery of sparse signals with support $\left\{t_{k}\right\}_{k=0}^{K-1} \in[0, \tau)$ at arbitrary points on the real line rather than restricted to a predescribed grid. Hence this leads to so-called "off-the-grid" recovery approaches [8].

The sparse signal recovery problem is closely tied to the topic of Shannon's sampling theory [9]. In analogy to the sampling of bandlimited signals where by the signal is pre-filtered with an anti-aliasing or low-pass filter, the measurements $g_{n}$ can be written as,

$$
g_{n}=\underbrace{\int s_{K}(t) \bar{\psi}(t-n T) d t}_{\text {Projection }} \equiv \underbrace{\left.\left(s_{K} * \psi\right)(t)\right|_{t=n T}}_{\text {Pre-filtering and Sampling }},
$$



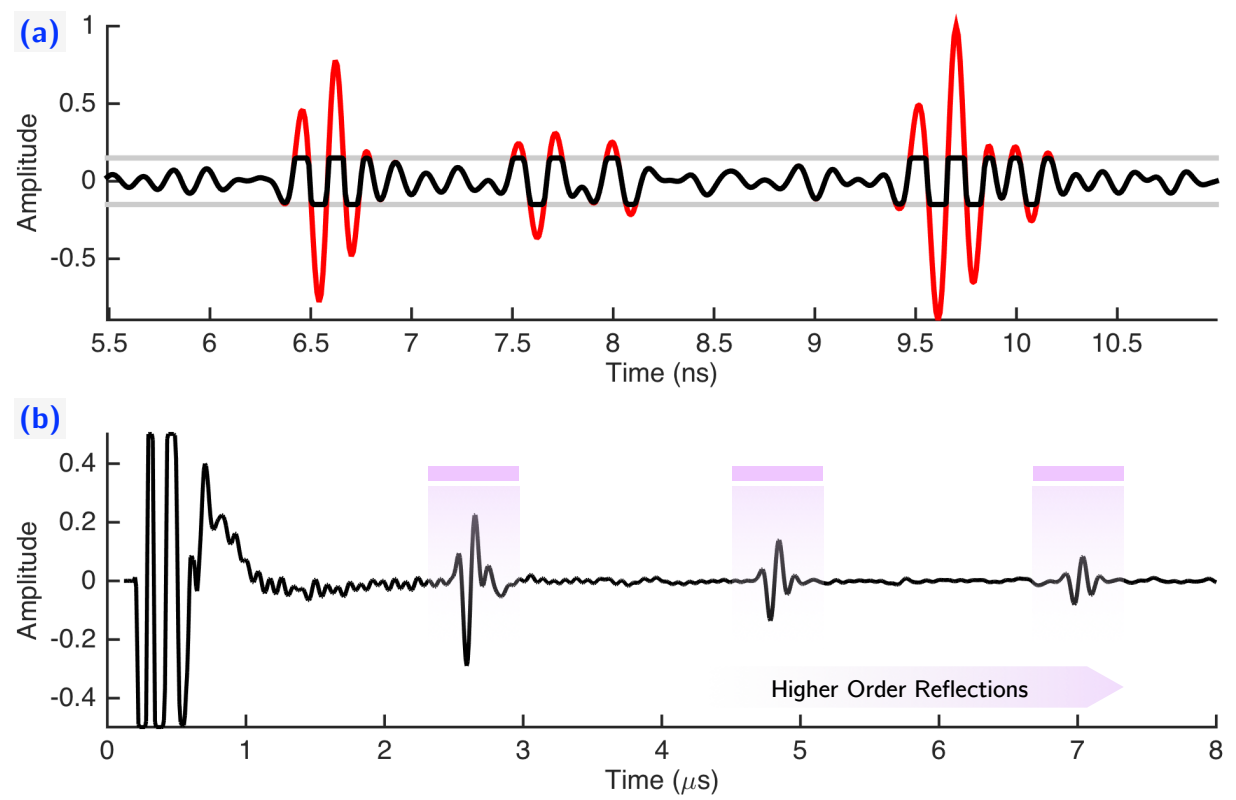

Fig. 1: Two practical scenarios for amplitude limited sampling. (a) Ultra-wide band signal undergoes saturation. (b) Data from ultrasonic sensor reveals that the dominant reflection is clipped or saturated as it exceeds the maximum recordable voltage of the ADC. In this case, exact calibration of $\psi$ is not possible.

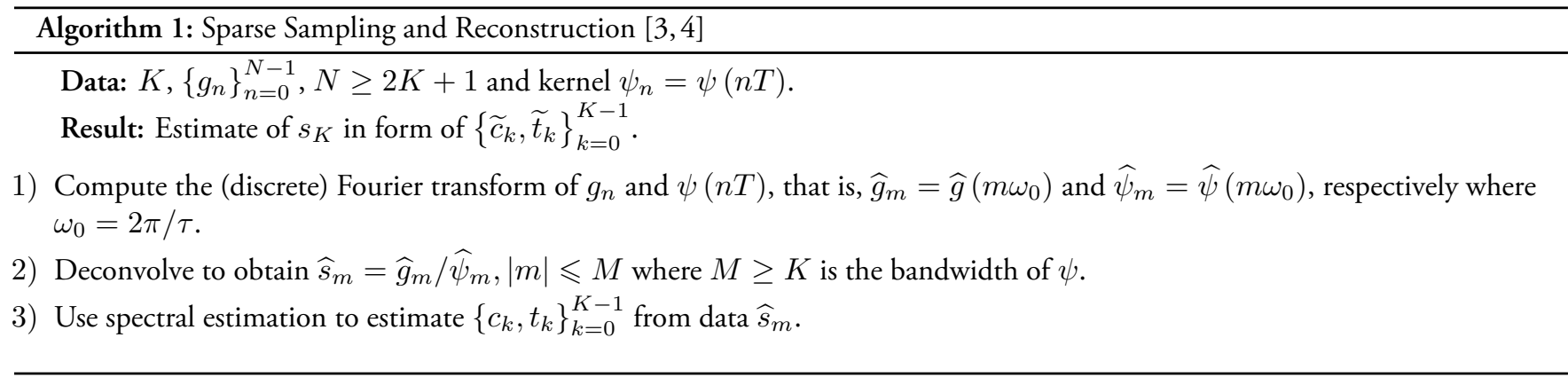

which is equivalent to low-pass projections of $s_{K}$ onto subspace of bandlimited function $\mathrm{V}_{\mathrm{BL}}=\operatorname{span}\{\bar{\psi}(t-n T)\}_{n=0}^{N-1}$ and where $\bar{\psi}(t)=\psi(-t)$. A natural question then is: When is the mapping between the sparse signal $s_{K}(t)$ and samples $\left\{g_{n}\right\}_{n=0}^{N-1}$, one-toone? It was shown by Li and Speed (cf. Thm 3.2, [2]) and Vetterli, Blu and co-workers (cf. Thm 1, [3], [4]) that $N \geq 2 K+1$ gurantees exact recovery of $s_{K}(t)$ from $g_{n}$ provided that the support or the locations $t_{k} \in[0, \tau)$ are distinct. The recovery procedure [3] then relies on Fourier domain extrapolation which is outlined in Algorithm 1.

The ability to sample and reconstruct sparse signals has found many applications including radio-astronomy [10], channel estimation [11], optical tomography [12], ultrasound imaging [13] and more recently, time-of-flight imaging [14, 15]. In view of (1), typically, bandlimitedness is defined in the Fourier domain; however, there are advantages of considering other unitary transforms. To this end, the recovery procedure has also been studied for the case of spherical harmonics [16,17], the Gabor transform [18] and generalizations of the Fourier transform [19,20].

\subsection{Sampling and Recovery of Sparse Signals in Practice}

In recovering sparse signals from low-pass projections, one fundamental assumption that is made in theory is that the dynamic range of the sensor or the analog to digital converter (ADC) is infinite. To the best of our knowledge, such assumptions appear in all previous works on the problem $[2,3,5,10-15]$.

In practice, however, $\mathrm{ADCs}$ are finite dynamic range devices and whenever a signal crosses the threshold (or the maximum recordable voltage), the measurements are saturated or clipped. Clipping of a bandlimited signal results in discontinuities which manifest as aliasing due to high frequency distortion in the Fourier domain [21]. In view of this, a number of numerical methods have been proposed in the literature [22-25], however, the exact link to sampling theory of bandlimited or sparse signals remains 


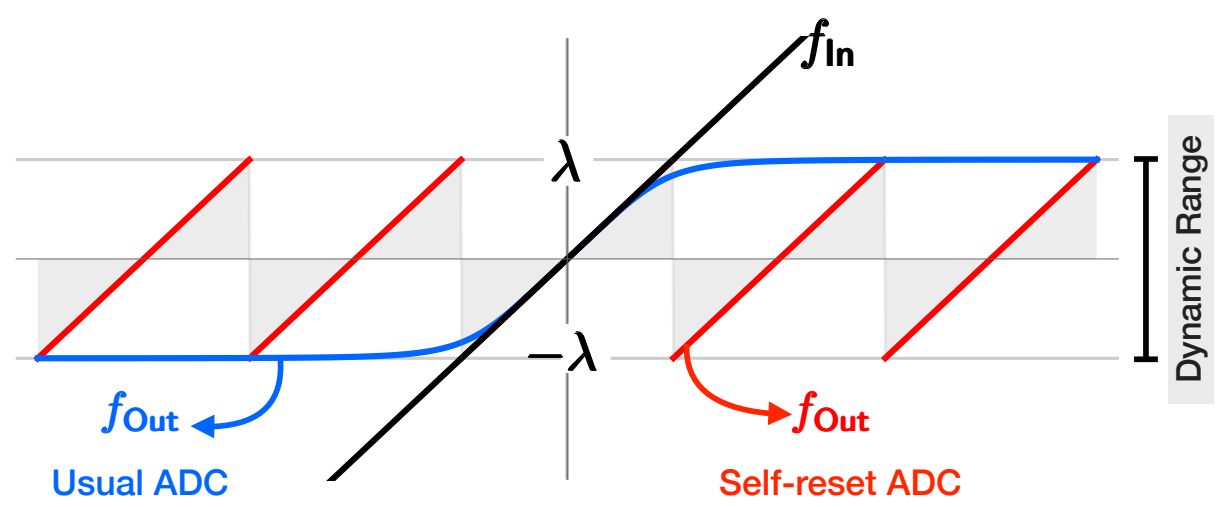

Fig. 2: Transfer function of conventional ADC compared with self-reset ADC. For conventional ADCs, whenever $\left|f_{\mathrm{In}}\right|>\lambda$, ADC saturates to $\lambda$ and this results in clipping. In contrast, whenever $\left|f_{\ln }\right|>\lambda$, the self-reset ADC folds $f_{\ln }$ such that $f_{\text {Out }}$ is always in the range $[-\lambda, \lambda]$. In this way, the self-reset configuration circumvents clipping but introduces discontinuities.

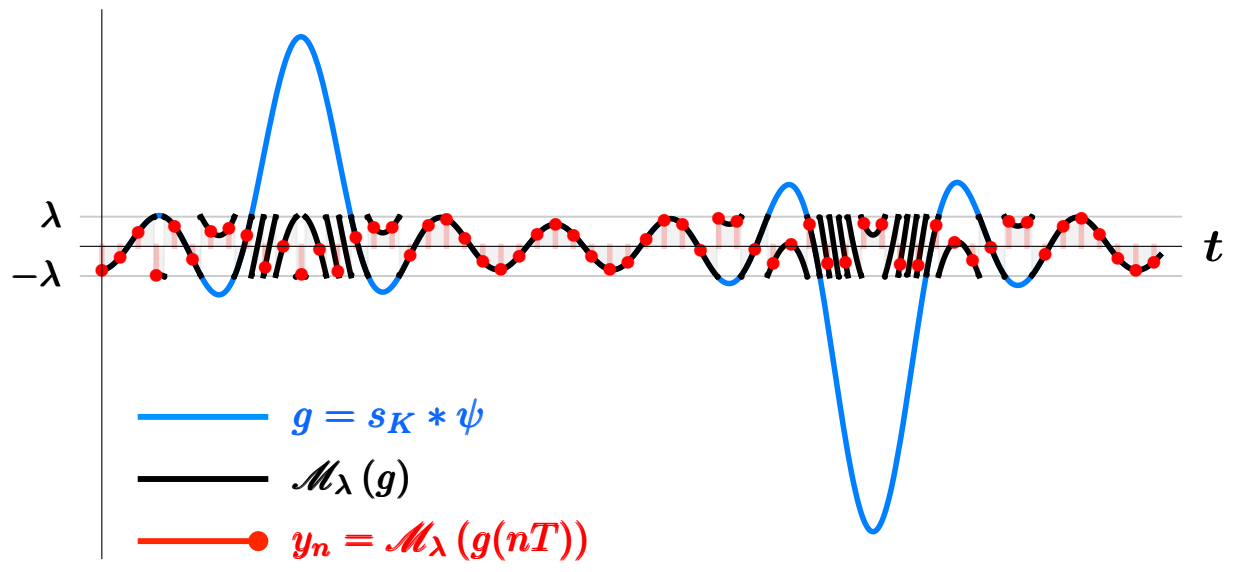

Fig. 3: Unlimited sampling of sparse signals with $K=2$. We plot low-pass filtered data $g$, the folded function $\mathscr{M}_{\lambda}(g)$ as well as modulo samples $y_{n}$ in $(5)$.

largely unclear.

This problem is of specific practical relevance in the context of calibration, namely, the knowledge of the unknown kernel $\psi$ is critical for accurate recovery of $s_{K}$ in sparse sampling models such as (1). In almost all of the applications, the kernel $\psi$ is obtained in a calibration phase [15].

During this phase, the received amplitudes are typically larger than during the following sensing phase, as shown via experimental measurements in context of ultra-wide band sensing in Fig. 1(a) and ultra-sonic non-destructive testing in Fig. 1(b). Consequently, either saturation limits the exact calibration of $\psi$ and the sparse sampling model (1) is invalid, or one has to work with a very high dynamic range, which will impact the measurement resolution as well as the penetration depth of $\psi$ (cf. $[15,26,27]$. In view of model (1), some application areas where this problem frequently arises includes ground penetrating radar [26] (cf. pg. 149, Fig. 5.2), seismic imaging [27], ultra-wideband sensing [28] and ultrasound imaging [29]. Not surprisingly, most of these solutions rely on:

1) ADC level corrections $[28,29]$, or,

2) De-clipping followed by deconvolution [27,30].

It is clear from literature that existing approaches decouple acquisition (hardware) from recovery algorithm (software). The downside being, hardware-only approaches $[28,29]$ are limited by computation that can be handled by hardware and algorithm-only approaches solve a sequential problem of de-clipping followed by spike recovery. For the latter, the quality of reconstruction depends on the effectivity of the de-clipping algorithm and is less attractive in practice because the $\psi$ may still be unknown.

\subsection{Our Contribution}

Our work is based on the recently introduced theory of "Unlimited Sampling" [1] which exploits a co-design between acquisition and recovery algorithm. On the acquisition front, we use self-reset ADCs (SR-ADCs) [31,32] which are based on a radically different 


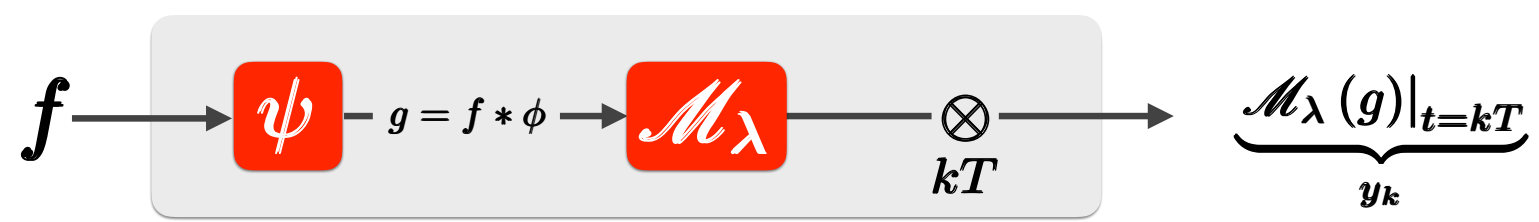

Data

Self-Reset ADC

Modulo Samples

Fig. 4: ADC architecture for Unlimited Sampling.

approach than the conventional ADCs in that whenever the input signal crosses a preset voltage threshold $\lambda$, the recorded voltage is reset (cf. Fig. 2). In this way, SR-ADCs allow for capturing voltages far beyond the saturation limit. The SR-ADCs rely on a folding architecture which is based on a memoryless, non-linear mapping [1],

$$
\mathscr{M}_{\lambda}: t \mapsto 2 \lambda\left(\llbracket \frac{t}{2 \lambda}+\frac{1}{2} \rrbracket-\frac{1}{2}\right)
$$

where $\llbracket t \rrbracket \stackrel{\text { def }}{=} t-\lfloor t\rfloor$ defines the fractional part of $t$. The mapping in (4) ensures that amplitudes are folded in the range of $\mathscr{M}_{\lambda}$, that is, $[-\lambda, \lambda]$. By using SR-ADCs, we circumvent the problem of ADC level corrections $[28,29]$ or clipping. However, it remains to recover $s_{K}$ from the SR-ADC based samples.

Consequently, the contributions of this paper are two fold:

1) We take a first step towards formalization of a sampling theorem for sparse signals which leads to a sufficient condition for prefect recovery of $K$-sparse signals from SR-ADC samples.

2) Our sampling theorem is complemented by a constructive algorithm which describes a linear and stable recovery procedure.

\section{UNLIMITED SAMPLING OF BANDLIMITED SIGNALS}

Here, we briefly review the basic principles at the core of unlimited sampling theory introduced in [1]. In view of the SR-ADC architecture discussed in [31,32], we will use the setup in Fig. 4 for obtaining modulo samples. Here, a finite energy signal (notnecessarily bandlimited) is pre-filtered with a bandlimited kernel $\psi$. Let $\widehat{\psi}(\omega)=\int \psi(t) e^{-\jmath \omega t} d t$ define the Fourier Transform of $\psi$. We say $\psi$ is $\Omega$-bandlimited or,

$$
\psi \in \mathcal{B}_{\Omega} \Leftrightarrow \widehat{\psi}(\omega)=\mathbb{1}_{[-\Omega, \Omega]}(\omega) \widehat{\psi}(\omega) \text { and } \psi \in L_{2}
$$

where $\mathbb{1}_{\mathcal{X}}(t)$ is the indicator function on set $\mathcal{X}$. Here, the pre-filtering operation, that is $y=f * \psi$, ensures that $y \in \mathcal{B}_{\Omega}$. Due to the SR-ADC architecture, the filtered signal $g$ undergoes amplitude folding defined by (4) and results in $z=\mathscr{M}_{\lambda}(g)$. The non-linearly folded signal $z$ is then sampled via impulse modulation and results in modulo samples,

$$
y_{n} \stackrel{\text { def }}{=} z(n T)=\mathscr{M}_{\lambda}(g(n T)), n \in \mathbb{Z}
$$

where $T>0$ is the sampling rate. The question of recovering $g(t) \in \mathcal{B}_{\pi}$ from modulo samples $y_{n}, n \in \mathbb{Z}$ is discussed in our companion paper [1] and the main result takes form of the following theorem:

Theorem 1 (Unlimited Sampling Theorem [1]). Let $g(t) \in \mathcal{B}_{\pi}$ and $y_{n}=\left.\mathscr{M}_{\lambda}(g(t))\right|_{t=n T}, n \in \mathbb{Z}$ in (5) be the modulo samples of $g(t)$ with sampling rate $T$. Then, a sufficient condition for recovery of $g(t)$ from the $\left\{y_{n}\right\}_{n}$ up to additive multiples of $2 \lambda$ is,

$$
0<T \pi e \leq 1 / 2
$$

The fundamental difference between recovery of sparse signal in (2) with respect to the bandlimited case discussed in [1] is that we will working with finite number of samples. Of course we expect that $N$ will be larger than $2 K+1$ but the number of samples should still be finite. Consequently, in working with sparse signals, there is a trade-off between sampling rate $T$ and number of modulo samples $N$. While $T$ dictates the recovery conditions for unfolding $g_{n}, N$ defines the critical number of samples required for estimation of $s_{K}$ from $g_{n}$. 


\section{UNLIMITED SAMPLING OF SPARSE SIGNALS}

Let $\psi \in \mathcal{B}_{\pi}$ be a given low-pass filter and $s_{K}$ be defined in (2). Furthermore, let $\left\{y_{n}\right\}_{n=0}^{N-1}$ in (5) be the modulo samples of $g$ defined in (1). The purpose of this section is to study the perfect reconstruction condition which gurantees recovery of continuous-time sparse signal $s_{K}$ from modulo samples $y_{n}$.

Our basic strategy for recovering $s_{K}$ from $y_{n}$ can be summarized as,

$$
y_{n} \stackrel{\text { Unfolding }}{\longrightarrow} g_{n} \stackrel{\text { Sparse Recovery }}{\longrightarrow} s_{K}(t)
$$

This approach relies on extracting unfolded, contiguous sample sequence $g_{n}$ of size $2 K+1$ from which $s_{K}(t)$ is estimated using high-resolution frequency estimation [2-4]. To see this, we split the problem into two parts which are discussed subsequently.

\subsection{Localized Reconstruction from Unlimited Sampling}

Given $g \in \mathcal{B}_{\pi}$ and $y_{n}, n \in \mathbb{Z}$ in (5), the problem of recovering $g_{n}, n \in \mathbb{Z}$ was discussed in [1]. In this work, in contrast to [1], it suffices to recover a subset of $g_{n}$ with size $N=2 K+1$ rather than the full sequence, but we only have finitely many modulo samples at our disposal. This fundamentally different setup requires a new approach which we will develop in this paper. The first step towards that goal is the same as in [1]. Namely the following lemma, which summarizes Lemma 1 and Proposition 2 of [1], shows that higher order differences $\Delta^{L}$, i.e., repeated applications of the first-order difference defined by $(\Delta v)_{n}=v_{n+1}-v_{n}$, of the modulo samples $y_{n}$ allow for the reconstruction of the higher order differences of the original signal ${ }^{1}$.

Lemma 1. For $g \in \mathcal{B}_{\pi}$, set $g_{n}=g(n T), T \in \mathbb{R}^{+}$and assume that some bound $\beta_{g} \geq\|g\|_{\infty}$ is available. Furthermore, assume that $T \pi e \leq \frac{1}{2}$ and choose

$$
L=\left\lceil\frac{\log \lambda-\log \beta_{g}}{\log (T \pi e)}\right\rceil .
$$

Then the sequence $y_{n}=\mathscr{M}_{\lambda}\left(g_{n}\right)$ of modulo samples satisfies

$$
\Delta^{L} g_{n}=\mathscr{M}_{\lambda}\left(\Delta^{L} g_{n}\right)=\mathscr{M}_{\lambda}\left(\Delta^{L} y_{n}\right) .
$$

Consequently, finding an $L$-th order finite differences of the sequence $g_{n}$ just requires the corresponding $L$-th order finite differences of the sequence $y_{n}$ of modulo samples, which in turn can be constructed from $L+1$ subsequent samples of $y_{n}$. Due to the overlap in the samples used, finding some number $R$ of subsequent $L$-th order finite differences of the sequence $g_{n}$ requires $L+R$ subsequent samples of $y_{n}$.

It remains to reconstruct the sequence $g_{n}$ from its $L$-th order finite differences. As in [1], we invert each of the repeated finite difference operators sequentially, and the difficulty is that in each step, the inverse is only defined up to an additive constant. Given that the modulo samples are available, this ambiguity consists of even integer multiples of $\lambda$, and the right constants can be derived from boundedness properties of bandlimited functions (cf. [1]).

More precisely, note that $g \in \mathcal{B}_{\pi}$ can be uniquely decomposed as $g=\mathscr{M}_{\lambda}(g)+\varepsilon_{g}$ where $\varepsilon_{g}$ is a simple function, $\varepsilon_{g}(t)=$ $2 \lambda \sum_{\ell \in \mathbb{Z}} e_{\ell} \mathbb{1}_{\mathcal{D}_{\ell}}(t), e_{\ell} \in \mathbb{Z}$. With $y_{n}=\mathscr{M}_{\lambda}(g(n T))$ given, knowing $\varepsilon_{g}$ is equivalent to the knowledge of $g_{n}$. Due to highly structured form of $\varepsilon_{g}$, there is a strong restriction on the range of the same. Namely, we may enforce the amplitude restriction that $\Delta^{\ell-1} \varepsilon_{g} \in 2 \lambda \mathbb{Z}$ when applying the anti-difference operation defined by, $S:\left(a_{i}\right)_{i=1}^{\infty} \mapsto\left(\sum_{i^{\prime}=1}^{i} a_{i^{\prime}}\right)_{i=1}^{\infty}$. We obtain that

$$
\left(\Delta^{\ell-1} \varepsilon_{g}\right)_{n}=\left(\mathrm{S} \Delta^{\ell} \varepsilon_{g}\right)_{n}+\kappa_{(\ell)} a_{n}, \quad a_{n}=2 \lambda . \kappa_{(\ell)} \in \mathbb{Z} .
$$

Since constants are in the kernel of $\Delta$, this cannot be resolved any further for $\ell=1$, we can only estimate $\varepsilon_{g}$ up to multiple of $2 \lambda \mathbb{Z}$. For $\ell>1$, however, we can apply $\mathrm{S}$ again and estimate the unknown $\kappa_{(\ell)}, \ell=1, \ldots, L$. We obtain

$$
\left(\Delta^{\ell-2} \varepsilon_{g}\right)_{n}=\left(\mathrm{S}^{2} \Delta^{\ell} \varepsilon_{g}\right)_{n}+\kappa_{(\ell)}(\mathrm{S} a)_{n}+\kappa_{(\ell-1)} a_{n} .
$$

and, given that $(\mathrm{S} a)_{n}$ is growing linearly, all but one choice of $\kappa_{(\ell)}$ will yield a sequence that violates the supremum bound entailed by the prior knowledge of $\beta_{g}$. As shown in [1], a sufficient number of subsequent samples of $\Delta^{\ell} y$ to distinguish the feasible choice of $\kappa_{(\ell)}$ from the infeasible ones is $6 \frac{\beta_{g}}{\lambda}$, and hence the required number of subsequent samples of $g$ is bounded by $6 \frac{\beta_{g}}{\lambda}+L+1 \leq 7 \frac{\beta_{g}}{\lambda}+1$ to reconstruct one value of $g$ and $7 \frac{\beta_{g}}{\lambda}+N^{\prime}$ to reconstruct $N^{\prime}$ subsequent values (cf. discussion after Lemma 1).

\footnotetext{
${ }^{1} \mathrm{~A}$ similar observation has been made in the phase-unwrapping literature where the well known Itoh's condition [33] requires $\|\Delta y\|_{\infty}<\lambda$. However, this approach is highly restrictive for it works only with $L=1$ and by inverting the discrete difference without exploiting any signal structure.
} 
Theorem 2 (Local Reconstruction Theorem). Let $g(t) \in \mathcal{B}_{\pi}$ with $\|g\|_{\infty} \leq \beta_{g}$ and $y_{n}=\left.\mathscr{M}_{\lambda}(g(t))\right|_{t=n T}, n=0, \ldots, N-1$ in (5) be the modulo samples of $y(t)$ with sampling rate $T$. Then a sufficient condition for recovery of $N^{\prime}$ contiguous samples of $g$ from the $y_{n}$ (up to additive multiples of $2 \lambda$ ) is that

$$
T \leq \frac{1}{2 \pi e} \text { and } N \geq N^{\prime}+7 \frac{\beta_{g}}{\lambda} .
$$

\subsection{A Sufficiency Condition for Recovering Sparse Signals}

To apply this theorem to the case of sparse sampling, recall that the number of subsequent samples required for reconstruction is $2 K+1$, which should hence also be our choice for $N^{\prime}$. Also note that using Young's inequality, one can bound

$$
\|g\|_{\infty}=\left\|s_{K} * \psi\right\|_{\infty} \leq\|\psi\|_{\infty}\left\|s_{K}\right\|_{\mathrm{TV}}
$$

where the $\|\cdot\|_{\mathrm{TV}}$ denotes the total variation of a measure, which, for spike trains, corresponds to the $\ell_{1}$-norm of the coefficient sequence $c_{k}$ in (2). Thus we obtain the following main result.

Theorem 3 (Unlimited sampling of sparse signals). Let $g=s_{K} * \psi$ for a known low-pass filter $\psi \in \mathcal{B}_{\pi}$ and $s_{K}$ in (2) be the unknown $K$-sparse signal to be recovered, and assume one has access to an a priori bound $\beta_{g} \geq\|\psi\|_{\infty}\left\|s_{K}\right\|_{\mathrm{TV}}$. Let $y_{n}=\left.\mathscr{M}_{\lambda}(g(t))\right|_{t=n T}, n=$ $0, \ldots, N-1$ in (5) be the modulo samples of $y(t)$ with sampling rate $T$. Then a sufficient condition for recovery of $s_{K}$ from the $y_{n}$ (up to additive multiples of $2 \lambda$ ) is that

$$
T \leq \frac{1}{2 \pi e} \text { and } N \geq 2 K+1+7 \frac{\beta_{g}}{\lambda} .
$$

Provided that this sufficiency condition is satisfied, and assuming that $\beta_{g}$ is known, by choosing $L$ prescribed by Lemma 1, Algorithm 2 recovers the sparse signal $s_{K}(t)$ from modulo samples $\left\{y_{n}\right\}_{n=0}^{N-1}$.

In contrast to [1] where the sampling bound is independent of SR-ADC threshold $\lambda$, in case of sparse sampling note that $N \propto \lambda^{-1}$. Since we are dealing with finite number of samples, this result is intuitive and we do expect that the number of samples required for sparse recovery will depend on both the sparsity level $K$ and the dynamic range $\beta_{g} / \lambda$ of the signal $g=s_{K} * \psi$.

\subsection{Numerical Demonstration}

We set up a numerical example where we set $K=3$ and $\tau=10$ to define $s_{K}(t)$ using $\left\{c_{k}, t_{k}\right\}$ chosen arbitrarily. This immediately gives, $\beta_{g}=3.2511$. We then acquire low-pass filtered measurements using $\psi(t)=\operatorname{sinc}(t)$ which is clearly $\pi$-bandlimited or $\psi \in \mathcal{B}_{\pi}$. With $\lambda=1 / 4$ and modulo sampling rate $T=1 /(2 \pi e)-1 / 100$, we acquire modulo samples $y_{n}$ using (5). By using result of Lemma 1, we obtain $L=3$. Furthermore, in view of (12), we must have at least $N=99$ modulo samples for recovery of $2 K+1$ contiguous values of unfolded $g_{n}$. We plot the sparse signal, its low-pass filtered version and the resultant modulo samples in Fig. 5 (a). By using the localized recovery method developed in Algorithm 2, we estimate unfolded samples $\widetilde{g}_{n}$ which is exactly the same as $g_{n}$ (upto machine precision) and this is shown in in Fig. 5 (b). In this computation, we assume the knowledge of constant offset since $\widetilde{g}_{n}$ may only be estimated upto a constant ambiguity of $2 \lambda \mathbb{Z}$. The mean squared error between ground truth $g_{n}$ and its estimate $\widetilde{g}_{n}$ is noted to be $5.0401 e^{-34}$. By choosing any contiguous set of size $2 K+1$ of the $N=99$ samples of $\widetilde{g}_{n}$, we can use the approach developed in [3] to estimate $s_{K}$.

\section{CONCLUSION}

In this paper, we considered the problem of recovery of sparse signals from low-pass filtered measurements which are sampled using self-reset or folding ADCs [1,31,32]. This novel ADC architecture maps low-pass filtered samples into modulo samples and hence circumvents any clipping or saturation. Since modulo operation is a non-linear mapping, in this paper we developed a strategy for local reconstruction of bandlimited signals from modulo samples. This result allows us to combine previously known methods for sparse signal recovery that were introduced in [3]. Our key result describes a perfect recovery condition for estimating a $K$-sparse signal from a finite number of modulo measurements. We provide a sampling bound for both the sampling rate as well as the number of samples needed for estimation of a $K$-sparse signal. Our work raises some interesting questions for future. For example, we note that the number of modulo samples depends on sparsity $(K)$ and the dynamic range of the signal $\left(\beta_{g} / \lambda\right)$. Through numerical experiments we empirically observed that our bound can be further sharpened. Furthermore, spike trains are a particular class of parametric signals. In future, we hope to develop results for a wider class of parametric signals. 

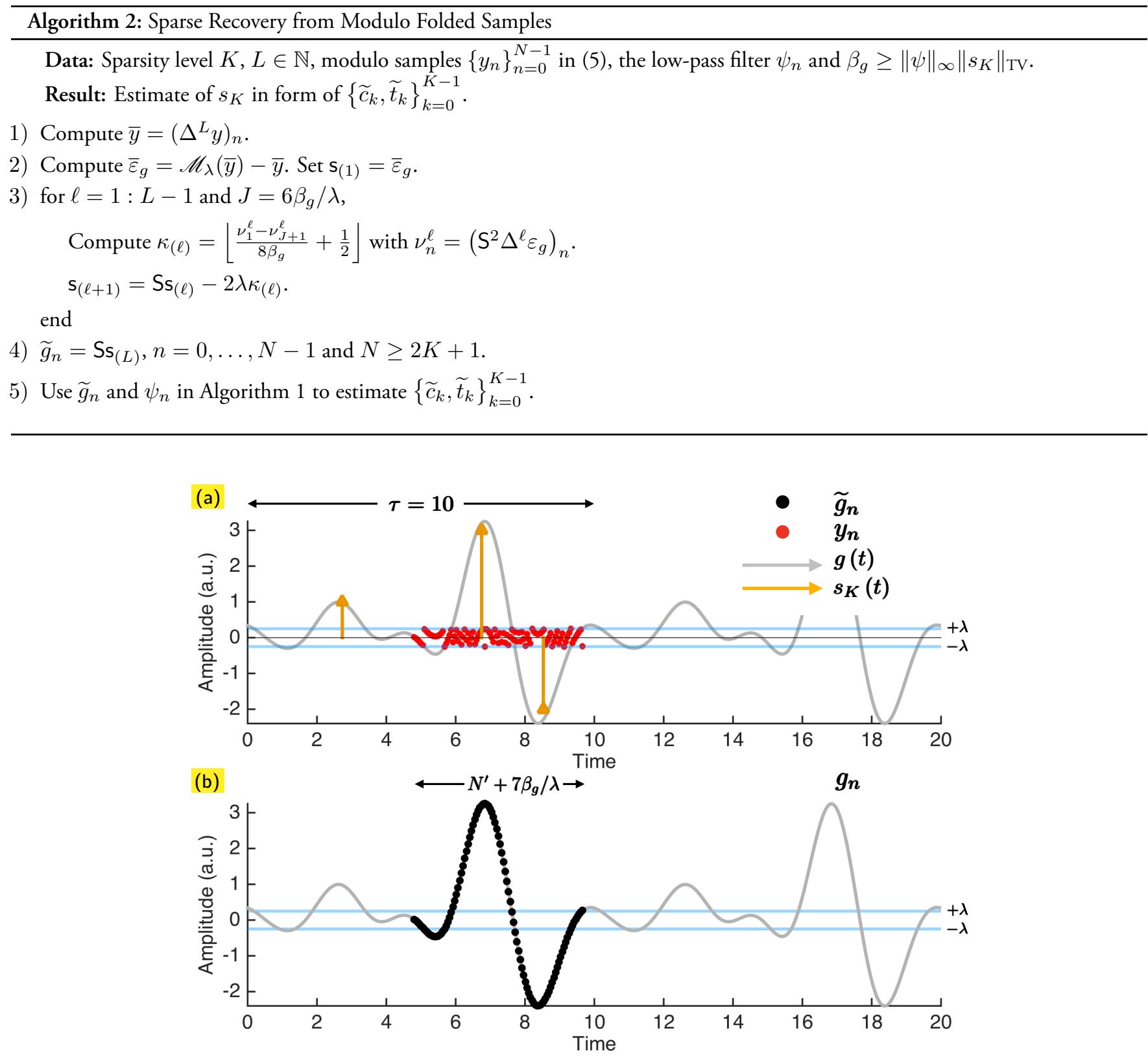

Fig. 5: Sparse signal recovery via local reconstruction of modulo samples with $\beta_{g}=3.2511$ and $\lambda=0.25$. (a) We plot $K$-sparse signal $s_{K}(t)$ with $K=3$ and $\tau=10$, the low-pass filtered signal $g=s_{K} * \psi$ where $\psi(t)=\operatorname{sinc}(t)$ as well as modulo samples $y_{n}=\mathscr{M}_{\lambda}\left(g_{n}\right)$ with $T=0.0485$. Note that $\psi \in \mathcal{B}_{\pi}$. (b) Using Algorithm 2, we estimate unfolded samples $\widetilde{g}_{n}$ from $N=99$ modulo samples of $y_{n}$. For this purpose $L=3$. The reconstruction is observed to be exact (upto machine precision). Given $2 K+1$ of $\widetilde{g}_{n}$, the spikes are estimated using Algorithm 1.

\section{REFERENCES}

[1] A. Bhandari, F. Krahmer, and R. Raskar, "On unlimited sampling," in Intl. Conf. on Sampling Theory and Applications (SampTA), Jul. 2017, pp. 31-35.

[2] L. Li and T. P. Speed, "Parametric deconvolution of positive spike trains," Annals of Statistics, pp. 1279-1301, 2000.

[3] M. Vetterli, P. Marziliano, and T. Blu, "Sampling signals with finite rate of innovation," IEEE Trans. Signal Process., vol. 50, no. 6, pp. $1417-1428,2002$.

[4] T. Blu, P. L. Dragotti, M. Vetterli, P. Marziliano, and L. Coulot, "Sparse sampling of signal innovations," IEEE Signal Process. Mag., vol. 25, no. 2, pp. 31-40, 2008. 
[5] E. J. Candès and C. Fernandez-Granda, "Towards a mathematical theory of super-resolution," Communications on Pure and Applied Mathematics, vol. 67, no. 6, pp. 906-956, Apr. 2013.

[6] S. Levy and P. K. Fullagar, "Reconstruction of a sparse spike train from a portion of its spectrum and application to high-resolution deconvolution," Geophysics, vol. 46, no. 9, pp. 1235-1243, Sep. 1981.

[7] F. Santosa and W. W. Symes, "Linear inversion of band-limited reflection seismograms," SIAM Jour. on Sci. and Stat. Computing, vol. 7 , no. 4, pp. 1307-1330, Oct. 1986.

[8] G. Tang, B. N. Bhaskar, P. Shah, and B. Recht, “Compressed sensing off the grid," IEEE Trans. Inf. Theory, vol. 59, no. 11, pp. 7465-7490, Nov. 2013.

[9] Y. C. Eldar, Sampling Theory: Beyond Bandlimited Systems. Cambridge University Press, 2015.

[10] H. Pan, T. Blu, and M. Vetterli, "Towards generalized FRI sampling with an application to source resolution in radioastronomy," IEEE Trans. Signal Process., vol. 65, no. 4, pp. 821-835, Feb. 2017.

[11] Y. Barbotin, A. Hormati, S. Rangan, and M. Vetterli, "Estimation of sparse MIMO channels with common support," IEEE Trans. Commun., vol. 60, no. 12, pp. 3705-3716, dec 2012.

[12] C. S. Seelamantula and S. Mulleti, "Super-resolution reconstruction in frequency-domain optical-coherence tomography using the finite-rate-of-innovation principle," IEEE Trans. Signal Process, vol. 62, no. 19, pp. 5020-5029, Oct. 2014.

[13] R. Tur, Y. C. Eldar, and Z. Friedman, "Innovation rate sampling of pulse streams with application to ultrasound imaging," IEEE Trans. Signal Process., vol. 59, no. 4, pp. 1827-1842, Apr. 2011.

[14] A. Bhandari and R. Raskar, "Signal processing for time-of-flight imaging sensors," IEEE Signal Process. Mag., vol. 33, no. 4, pp. 2-16, Sep. 2016.

[15] A. Bhandari, A. M. Wallace, and R. Raskar, "Super-resolved time-of-flight sensing via FRI sampling theory," in IEEE Intl. Conf. on Acoustics, Speech and Signal Processing, Mar. 2016.

[16] S. Deslauriers-Gauthier and P. Marziliano, "Sampling signals with a finite rate of innovation on the sphere," IEEE Trans. Signal Process., vol. 61, no. 18, pp. 4552-4561, 2013.

[17] I. Dokmanić and Y. M. Lu, “Sampling sparse signals on the sphere: Algorithms and applications,” IEEE Trans. Signal Process., vol. 64, no. 1, pp. 189-202, Jan. 2016.

[18] C. Aubel, D. Stotz, and H. Bölcskei, "A theory of super-resolution from short-time fourier transform measurements," Journal of Fourier Analysis and Applications, 2017.

[19] A. Bhandari and P. Marziliano, "Sampling and reconstruction of sparse signals in fractional Fourier domain," IEEE Signal Process. Lett., vol. 17, no. 3, pp. 221-224, 2010.

[20] A. Bhandari and Y. C. Eldar, "A Swiss army knife for finite rate of innovation sampling theory," in IEEE Intl. Conf. on Acoustics, Speech and Signal Processing, Mar. 2016, pp. 3999 - 4003.

[21] F. Esqueda, S. Bilbao, and V. Valimaki, “Aliasing reduction in clipped signals," IEEE Trans. Signal Process., vol. 64, no. 20, pp. 5255-5267, Oct. 2016.

[22] J. Abel and J. Smith, "Restoring a clipped signal," in Proc. of IEEE ICASSP, 1991.

[23] S.-K. Ting and A. H. Sayed, "Mitigation of clipping in sensors," in Proc. of IEEE ICASSP, May 2013.

[24] A. Adler, V. Emiya, M. G. Jafari, M. Elad, R. Gribonval, and M. D. Plumbley, "A constrained matching pursuit approach to audio declipping," in Proc. of IEEE ICASSP, May 2011.

[25] J. Zhang, J. Hao, X. Zhao, S. Wang, L. Zhao, W. Wang, and Z. Yao, "Restoration of clipped seismic waveforms using projection onto convex sets method," Nature Scientific Reports, vol. 6, no. 1, Dec. 2016.

[26] N. J. Cassidy, Ground Penetrating Radar Theory and Applications. Elsevier Science, 2009, ch. Ground Penetrating Radar Data Processing, Modelling and Analysis, pp. 141-176.

[27] W. Yang and Y. Ben-Zion, "An algorithm for detecting clipped waveforms and suggested correction procedures," Seismological Research Letters, vol. 81, no. 1, pp. 53-62, Jan. 2010.

[28] C. Anderson, S. Venkatesh, J. Ibrahim, R. Buehrer, and J. Reed, "Analysis and implementation of a time-interleaved ADC array for a software-defined UWB receiver," IEEE Trans. Veh. Technol., vol. 58, no. 8, pp. 4046-4063, Oct. 2009.

[29] M. Kaplan, A. Lundberg, and T. M. Duffy, "Ultrasound imaging system apparatus and method with ADC saturation monitor," U.S. Patent US8 568319 B1, Oct., 2013.

[30] T. Olofsson, "Deconvolution and model-based restoration of clipped ultrasonic signals," IEEE Trans. Instrum. Meas., vol. 54, no. 3, pp. 1235-1240, Jun. 2005.

[31] J. Rhee and Y. Joo, “Wide dynamic range CMOS image sensor with pixel level ADC,” Electron. Lett., vol. 39, no. 4, p. 360 , 2003.

[32] K. Sasagawa, T. Yamaguchi, M. Haruta, Y. Sunaga, H. Takehara, H. Takehara, T. Noda, T. Tokuda, and J. Ohta, "An implantable CMOS image sensor with self-reset pixels for functional brain imaging," IEEE Trans. Electron Devices, vol. 63, no. 1, pp. 215-222, Jan. 2016.

[33] K. Itoh, "Analysis of the phase unwrapping algorithm," Applied Optics, vol. 21, no. 14, pp. 2470-2470, Jul. 1982. 